\title{
Two Cases of Spontaneous Mediumship Experiences of Near-Death Experiencers
}

\author{
Ryan D. Foster, $\mathrm{PhD}$ \\ Deborah Lee, BS \\ Ann Grau Duvall, BS \\ Marymount University
}

This article is dedicated to the life and memory of Deborah Lee.

ABSTRACT: Spontaneous mediumship experiences (SMEs), in which living people are visited uninvited by discarnates-deceased humans—-who ask the living person to convey a message to another living person, are considered a subtype of after-death communication and a potential aftereffect of near-death experiences. In this article, we describe two case studies based on semi-structured interviews in which two near-death experiencers described features and descriptions of their SMEs, positive and negative aspects associated with their SMEs, and related experiences including help-seeking behaviors. Implications for characterizing SMEs include differences in degree of spontaneity and types of discarnates and similarities in experiences of distress and number of SMEs.

KEY WORDS: spontaneous mediumship experience, after-death communication, near-death experience aftereffects

Near-death experiencers (NDErs) report a number of aftereffects that reflect post-NDE changes in their lives in a variety of domains, includ-

\footnotetext{
Ryan D. Foster, PhD, LPC, NCC, CHST, is assistant professor in the Department of Counseling at Marymount University. Reverend Deborah Lee, BS, passed away on December 23, 2015, less than a week after completing revisions on this article. She was active in a spiritual community in the Mid-Atlantic region of the United States and devoted her life to providing pastoral and counseling service to others. At the time of the study described in this article, she was a master's student in the Pastoral Clinical Mental Health Counseling Program, and at the time of her passing she was a doctoral student in the Counselor Education and Supervision Program at Marymount University. Ann Grau Duvall, BS, was a master's student in the Pastoral Clinical Mental Health Counseling Program at Marymount University at the time of this study, and is now a Qigong facilitator at Peaceable Dragon in Northern Virginia. Correspondence regarding this article should be sent to Dr. Foster at 2807 N. Glebe Road, Arlington, VA 22207; e-mail: rfoster@marymount.edu.
} 
ing intrapersonal, interpersonal, environmental, and transpersonal (Noyes, Fenwick, Holden, \& Christian, 2009). Ever since Raymond Moody's (1975/2001) seminal qualitative descriptions, NDE investigators and researchers have worked to identify, report, and contextualize the many aftereffects NDErs experience, and the literature is replete with narrative after narrative of, at this point, familiar postNDE experiences. However, one aftereffect, termed spontaneous mediumship experience (SME) by Holden, Foster, and Kinsey (2014), is in its infancy of scientific investigation. In SME, a person experiences the presence of an uninvited discarnate-a deceased human-who asks the experiencer to convey a message to another living person who is an intimate of the discarnate and whom the experiencer may or may not know. We direct readers to Holden et al. (2014) for a more detailed explanation of SMEs, including how they differ from typical after-death communication (ADC) experiences.

Mediumship is well documented but has no materialist scientific explanation. Generally speaking, mediumship is an experience in which a living person, a medium, acts as a mode of communication through which a discarnate communicates a message to yet another living person, termed the sitter (Beischel, 2015). Most non-SME mediumship experiences are intentional, whereas very few are spontaneous (Guggenheim \& Guggenheim, 1995; Knight, 2011; LaGrand, 1997). Further differentiating SMEs from other mediumship experiences is the relationship between the medium and the sitter. According to Holden et al. (2014),

a traditional medium typically knows the sitter, whereas a spontaneous medium may not know the living person for whom the message is intended; even if the person is known, that person typically did not seek a message from the discarnate. (pp. 70-71)

Although a few authors described experiences similar to SMEs in investigations of ADC (e.g., Guggenheim \& Guggenheim, 1995; Knight, 2011; LaGrand, 1997), the work of Holden et al. (2014) is the only formal empirical study yet to focus solely on this particular transpersonal experience among NDErs.

Holden et al. (2014) performed an exploratory mixed-methods study in which they reported their quantitative findings. In their survey of 89 NDErs, they found that $41.6 \%$ of participants had at least one SME after their first or only NDE. A majority of participants (47\%) reported little to no distress associated with their SMEs, but one-quarter (25\%) reported moderate to extreme distress. Ultimately, Holden et al. sug- 
gested a number of strategies that mental health providers could use when counseling an NDEr that complement the approach that I (RDF) and my co-authors described previously (Foster, Holden, \& James, 2009), primarily with a psychoeducational approach as to the nature of some aftereffects including SMEs. Naturally, a suggested implication of the Holden et al. (2014) study is that qualitative approaches to research could assist in delineating experiential nuances of SMEs in ways that quantitative methodology cannot. Thus, the purpose of the current article is to report two case studies of NDErs who reported having had SMEs. The intent of examining these case studies is to gain a deeper understanding and add to an emerging characterization of SMEs.

\section{Method}

During the recruitment and data collection phase of a prior study (Holden et al., 2014), two participants subsequently contacted RDF via email to inquire about SMEs. Both participants shared with me that they had experienced NDEs and SMEs, and they asked if I would be interested in talking with them further about their experiences due to their interest in furthering research understanding of SMEs. I told them that I was interested but that before we could proceed, I would have to gain Institutional Review Board (IRB) approval through my university, and they understood. After gaining approval from the Marymount University IRB, I contacted the participants and evaluated their current interest in proceeding with an interview with one of my research assistants. They both agreed, and I mailed them the informed consent form along with a stamped return envelope, which I received back from them.

Subsequently, the other two authors of this study, who were trained research assistants (RAs) and master's-level students in a pastoral clinical mental health counseling program at the time of this study, each contacted one of the participants to schedule a phone interview. The semi-structured interviews were based on the survey questionnaire used in the Holden et al. (2014) study, with questions modified to be open-ended rather than forced-choice (see Appendix). Both RAs audio recorded the interviews and transcribed them. Following are summaries of each narrative in which participant names and some details have been changed to ensure confidentiality. 


\section{The Case of Alexandria}

At the time of her interview, Alexandria was a 51-year-old white female with a graduate degree in a hard science field. She reported three NDEs: a pleasurable one associated with a childhood accident at age 2 years, a distressing one associated with a burst appendix at age 10 , and a pleasurable one associated with a car accident at age 29 . Alexandria's SMEs began happening after her first NDE and were a common experience; at that time, she labeled them as "imaginary friends;" her parents were aware of them and couched them as normal childhood experiences. However, Alexandria's SMEs as a young child also included, for example, seeing her grandfather's deceased fellow soldiers with whom he had fought in World War II.

Alexandria reported that between her first and second NDEs her SMEs occurred frequently and consistently. As a child and into adolescence, she believed SMEs were a normal human experience. After her second NDE her SMEs diminished quite a bit but then increased again after her third NDE. She reported that since her third NDE her SMEs have been too numerous to count.

Alexandria's experiences of seeing and communicating with discarnate entities have rarely been distressing. One exception was a time when she had an argument with her deceased ex-husband. Other exceptions, which she considers her most distressing SMEs, have been the occasional times when discarnates have asked her to pass messages along to other living persons:

Because my fears have always been if I share certain experiences, people are going to call me a fraud, or they say you are going to trick me, or you are delusional. You are either stupid for believing this stuff, or delusional for thinking that you are having these experiences, or just cheating us somehow.

One notable distressing aftermath of SMEs came as a result of an experience with one of her longtime colleagues. Alexandria was having a professional meeting via Skype with her friend, Jerry, and she saw a young adult male behind him wearing scrubs-who she believed was another living person. She asked Jerry about the person in the room, and Jerry stated that there was no one in the room but asked Alexandria to describe him. The discarnate male gave his name, and she passed this information on to Jerry. Jerry told Alexandria that his son, a medical student, had recently died-unbeknownst to Alexandria-and his nickname was the one that Alexandria passed on to him. 
Subsequently, during regular professional meetings with Alexandria, Jerry would repeatedly ask her to "do the medium thing." Mediumship was a spontaneous experience for Alexandria but not one that she enjoyed. She had no desire or intention to practice or share the experience. Jerry, in his excitement, became almost obsessive in his insistence that Alexandria pass on messages. The focus was no longer on their research but on Jerry's desire to be in communication with his son. Alexandria's great discomfort with communicating mediumship experiences "ended what had been a really good friendship up until then and a working relationship."

This experience reflects Alexandria's greatest fear with her SMEs. Communicating messages from discarnates to living persons may affect negatively her relationships with others:

In terms of what I pass on to others, and I rarely do, I mean the ghosts themselves are not frightening, typically. It's just people's reactions to them. Someone saying this is what I saw, that's where the distress comes in for me. That's why I don't usually pass on [information I receive]. I will try and find ways around passing on messages like [by] bringing up topics and talking about something that is brought up without actually saying, "This is your dead [acquaintence's] information."

In other words, Alexandria worried that others may think she is crazy or hallucinating if she were to reveal that she communicates with discarnates. Another challenging aspect of Alexandria's SMEs is that she "can't always tell when [the entities she is perceiving] are alive or dead" because they appear visually no different from living people; therefore, she cannot make any obvious initial distinctions. Therefore, she is fearful of responding to people who engage with her whom she does not personally recognize, with other living people around, out of fear that she may be communicating with a discarnate and that others will view her as mentally unhealthy.

However, Alexandria has also had positive experiences with communicating SME messages. One such experience happened over the course of several months with the most recent incident occurring just days prior to her interview. While participating in a study on meditation, Alexandria was visited by a discarnate male who identified himself as Hank. She reported her experiences to investigators leading the study, and they encouraged her to continue passing on the messages Hank was communicating to her. Alexandria's experiences with Hank were "comforting," and as her experiences multiplied, investigators stated that they believed they knew Hank. Hank was a 
popular man in the area where they lived and was locally known for some of his research work. Additionally, acquaintances of hers knew Hank and encouraged her to contact Hank's living wife. After much consideration, Alexandria telephoned Hank's wife and had a lengthy and confirming conversation.

In the past four years, Alexandria began to understand her paranormal experiences in a new way that has led to normalization of both her NDEs and SMEs. She has read literature, met with parapsychologists, and participated in research studies, which have all been positive psychoeducational experiences. She has also attended conferences of the International Association for Near-Death Studies (IANDS) where she interacted with other transpersonal experiencers, about half of whom have experienced an NDE. As a result, she understands now that her NDEs and SMEs are part of the trajectory of normal human experiences, and that knowledge has enabled her to move toward acceptance and integration of the experiences.

\section{The Case of Beth}

At the time of her interview, Beth was a 50-year-old white female with some college education. She had her first and only NDE at age 43 years: While in hospital being treated for a migraine headache, the staff accidentally administered a medication overdose, during which time Beth had her NDE. She stated that post-NDE, she has had at least 100 SMEs. In addition to SMEs, she also reported the common NDE aftereffect whereby electromagnetic devices malfunction in the NDEr's vicinity (Nouri \& Holden, 2008).

At the time of the interview, Beth had been keeping a journal of her SMEs to help in her organization and understanding of her experiences. It was not always that way: Initially, discarnates would come to her, and she neither understood the experiences nor felt any control over them. However, she reported that over time she began writing everything down so that she could keep track and feel more organized. She even learned to set temporal boundaries, for example, communicating to discarnates a specific time, convenient for her, when they should come back for a session. She sometimes has provided mediumship sessions for living people who requested them. In these cases, she asked specific questions, such as discarnates' names, their home cities and states, and a basic description of them. She avoids having any other specific information so that she can be sure that she is recording the information given by the discarnate rather than what she was told 
by the living person. Regarding the subjective, conscious experiences of her SMEs, Beth reported that communication sometimes has been verbal and other times has involved colors, impressions, drawings, and smells - the latter a sure indicator to Beth of an SME, as she lost her olfactory sense years ago as a result of a surgery.

Beth identified several challenges and distressing aspects of her SMEs. Most of her SMEs have involved victims and perpetrators of violent crimes. Beth's SMEs have often helped other living persons; however, at times she has experienced victims' pain as she communicated with them. Understandably, she found such SMEs physically and emotionally draining, as in the case of a discarnate male who entered her body and apologized to his daughter for molesting her as a child. Beth reported that after this experience, she has no longer allowed discarnates to come into contact with her body. She maintains this boundary by verbally telling the discarnate not to touch her. In some cases, when the discarnate has been so excited that she was unable to communicate with her or him, she sent the discarnate away with an invitation to return when she or he had calmed down enough to honor Beth's boundaries.

In general, Beth's experiences with discarnates have stopped being a source of distress to her because she has, to a great degree, learned to control them. Similar to Alexandria, however, Beth has felt distress as a result of communicating messages to living persons.

One example of this type happened while Beth was a waitress. One day, she decided to have a glass of wine at the restaurant at which she waitressed. She noticed a man she did not know sitting next to her, and she suddenly asked if it was his birthday, to which the man responded affirmatively. She told him, "I kind of have this lady tapping on my shoulder, wanting me to tell you happy birthday." He showed interest in Beth's message and asked her to continue. Beth communicated to him that he should go home and look in two particular places for something important. After telling him a few other messages, the man began to cry and left the restaurant. Beth became concerned about him and worried that she would lose her job because she upset a customer.

The next day, the man came back and thanked her. He reported to her that he had been feeling suicidal, and after looking for the two objects, his suicidality disappeared. The two objects he found were a sobriety pin and the obituary of his wife. According to the man, his wife had had a terminal illness during which the man rarely left her hospital bedside. The moment that she died was during a brief time 
when the man left her side to get a cup of coffee; subsequently, he had felt intense depression and guilt for what felt to him like abandoning her to die alone. He interpreted the messages as his wife's feelingnot of abandonment but of relief that he was not there when she died: "I'm relieved that you weren't in the room, because it's ugly. I didn't really want you there for that." Thus, rather than feeling abandoned, she felt gratified to have spared him the unnecessary suffering of witnessing her last, apparently tortured, moments of life.

Another challenging aspect of Beth's SMEs is that she feels unable to use what she perceives as a gift from her NDE on a larger scale. She enjoys helping individual people but would like her SME abilities to benefit the world in a greater way.

Beth reportedly did not seek formal consultation with health professionals to help her to manage or cope with distressing aspects of her SMEs. She has had informal talks with a friend who has been helpful in normalizing her experiences, and she has read many books that she found helpful, as well. However, the most helpful process in dealing with her SMEs has been the validation she has experienced when she communicated messages to other living persons-validations that her SMEs are real and not imagined, and that she is not "crazy."

\section{Implications and Conclusions}

Individuals' NDEs vary in specific content and aftereffects, and based on these two case study reports, this variability appears to apply to specific content of SMEs. One major difference between Alexandria's and Beth's SMEs is the degree to which the experiences are spontaneous versus controlled: Alexandria's appear far more spontaneous compared to Beth's that she reported being able to organize and planalthough Beth continues to have spontaneous experiences. Another major difference is the types of discarnates who visit each person: Alexandria's run the gamut of discarnates' life and death circumstances, whereas Beth's include a large proportion of discarnates whose lives and/or deaths involved violation of some type, either as perpetrator or as victim.

In contrast, there are also a few similarities between these two cases. Both Alexandria and Beth have attempted, at least in part, to manage stress associated with SMEs through seeking validation and normalization from people in their personal lives and also through reading literature. Additionally, they both experienced some distress when communicating their messages to living people, especially the 
potential relational consequences. Finally, Beth and Alexandria identified having countless numbers of SMEs.

During both Alexandria's interview of one hour and Beth's of 90 minutes, each of these SMErs appeared to be in good mental health. The observations of their mental health statuses were made by their interviewers who were both advanced clinical mental health counseling interns with clinical training in mental health diagnosis and assessment. Alexandria has rarely been without the experience of SMEs since they began for her in childhood. Beth, although developing the ability later in life, has learned to accept and integrate them into her normal routine. The interviewers' observations of mental health are consistent with Jenny Streit-Horn (2011) who found that although no researcher had conducted a study specifically assessing the mental health of after-death communicators, several of the researchers of the 36 studies of ADC that had been published between 1894 and 2006 had commented that their interviewees appeared quite mentally healthy. Similarly, in a recent qualitative study of ADC for her doctoral thesis, social worker Michele Knight (2011) conducted multiple in-depth interviews with 21 bereaved adults in Sydney, Australia who, like she, had experienced one or more ADCs with deceased loved ones; she also affirmed their apparent mental health.

An obvious limitation to this study is the informal case study design. Although Alexandria's and Beth's experiences may be important anecdotal contributions to NDE researchers' understanding of SMEs, the findings of this study cannot be generalized to other NDErs who have had SMEs.

However, some data from this study appear to support and/or underscore results found elsewhere in the literature. Like the majority of participants in Holden et al.'s (2014) study, Alexandria and Beth marked the onset of their SMEs to have been in the aftermath of, and attributable to, their NDEs. Additionally, like the majority of participants in Holden et al.'s study, Alexandria and Beth found at least one helpful coping strategy. Finally, one notable result of the Holden et al. study was the amount of distress participants associated with their SMEs: A majority indicated that their SMEs were slightly to not at all distressful. In a way, these results seem to coincide with what Alexandria and Beth reported in terms of occasional distress but little distress overall associated with their communication with discarnates. However, passing along messages from discarnates to living loved ones was a major source of distress associated with Alexandria's 
and Beth's SMEs, and this specific aspect may not have been captured in Holden et al.'s survey.

Echoing Holden et al. (2014), we conclude from these two case studies that NDErs need to know more about the potential for SMEs. Healthcare providers, authors, NDE investigators, and organizations such as Friends of IANDS and IANDS itself should commit resources to offer information and support for NDErs who may experience SME-or at least convey the fact that SMEs are a potential aftereffect of NDEs. Additionally, it appears that SMErs may need supportive resources developed to assist them in strategically organizing and coping with their SMEs. Alexandria and Beth reported having numerous SMEs, and the differences between experiences like these and pathological experiences like hallucinations should be made clear to SMErs. For example, from Alexandria's and Beth's experience, information that discarnates pass on to SMErs has a degree of veridicality and verifiability. Alexandria and Beth both discussed that when they have passed on messages from discarnates to living persons, those living persons verified their relationships with the discarnates and the information passed along. This kind of verifiability is not the case with hallucinatory or delusional experiences (American Psychiatric Association, 2013). In addition, people who experience pathological forms of hallucinations and delusions often experience co-morbid or underlying mood disorders and other forms of psychopathology. Although it is unclear if SMErs were included in their sample, Greyson and Liester (2004), in their comparison of auditory hallucinations in non-NDErs and NDErs, noted that their results did not support psychopathology in their sample of NDErs-nor did we observe it in either SMEr, although neither participant in our study completed any objective measure of mental health.

Some potential implications for NDE-SME researchers emerged in this study. Future surveys or interviews with SMErs should include questions designed in a more nuanced fashion to inquire about distress associated with SMEs. Researchers should ask about distress associated with communicating with discarnates as well that associated with communicating messages to living persons. In addition, further NDE aftereffect research should include questions about SMEs in general to continue to add to understanding about this phenomenon. There is mounting evidence to support SMEs as a verifiable aftereffect of NDEs that may be more common and particularly consequential than has previously been addressed in the field of near-death 
studies. As a result of this continued data collection, further qualitative and quantitative studies will be important contributors not only to scientific understanding of SMEs but also to increased support and education for SMErs in their search to manage and integrate their experiences.

\section{References}

American Psychiatric Association (2013). Diagnostic and statistical manual of mental disorders (5th ed.). Washington, DC: Author.

Beischel, J. (2015). Investigating mediums: A Windbridge Institute collection. San Francisco, CA: Blurb.

Foster, R.D., James, D., \& Holden, J.M. (2009). Practical applications of research on near-death experiences. In J.M. Holden, B., Greyson, \& D. James (Eds.), The handbook of near-death experiences: Thirty years of investigation (pp. 235-258). Santa Barbara, CA: Praeger/ABC-CLIO.

Greyson, B., \& Liester, M.B. (2004). Auditory hallucinations following neardeath experiences. Journal of Humanistic Psychology, 44(3), 320-336.

Guggenheim, B., \& Guggenheim, J. (1995). Hello from heaven! New York, NY: Bantam.

Holden, J.M., Foster, R.D., \& Kinsey, L. (2014). Spontaneous mediumship experiences: A neglected aftereffect of near-death experiences. Journal of NearDeath Studies, 33(2), 69-85.

Knight, M. (2011). Ways of being: The alchemy of bereavement and communiqué (Doctoral thesis). Sydney, Australia: University of Sydney.

LaGrand, L. (1997). After death communication: Final farewells. Woodbury, MN: Llewellyn.

Moody, R. (2001). Life after life: The investigation of a phenomenon-survival of bodily death. San Francisco, CA: Harper. (Original work published 1975)

Nouri, F.M., \& Holden, J.M. (2008). Electromagnetic aftereffects of near-death experiences. Journal of Near-Death Studies, 27, 83-110.

Noyes, R., Fenwick, P., Holden, J.M., \& Christian, R. (2009). Aftereffects of pleasurable Western adult near-death experiences. In J. M. Holden, B. Greyson, \& D. James (Eds.), The handbook of near-death experiences: Thirty years of investigation (pp. 41-62). Santa Barbara, CA: Praeger/ABC-CLIO.

Streit-Horn, J. (2011). A systematic review of research on after-death communication $(A D C)$ (Unpublished doctoral dissertation). University of North Texas, Denton, TX. 


\section{Appendix}

NDE SME Semi-Structured Interview

\section{Section 1}

1. What is your sex?

2. What is your year of birth?

3. What is your ethnicity?

4. What is your education level?

5. When was/were your NDE(s)?

6. What were the circumstances surrounding your NDE(s)?

\section{Section 2}

For the purpose of this study, the experience of being visited by a deceased person who communicated a message they wanted you to convey to another living person is termed a "spontaneous mediumship experience" (SME).

7. Before your NDE, approximately how many SMEs have you had?

8. After your NDE, approximately how many SMEs have you had?

9. Of all the SMEs you indicated since your NDE, please identify the one that, for whatever reason(s), you consider the least distressing.

a. Please describe this experience in detail, including circumstances, contents, and aftermath.

b. What made this experience the least distressing one?

c. How distressing was the experience itself?

d. How distressing was the aftermath of the experience?

10. Of all the SMEs you indicated since your NDE, please identify the one that, for whatever reason(s), you consider the most distressing.

a. Please describe this experience in detail, including circumstances, contents, and aftermath.

b. What made this experience the most distressing one? 
c. How distressing was the experience itself?

d. How distressing was the aftermath of the experience?

11. Considering all the SMEs you have experienced since your first (or only) NDE, how distressing overall have you considered these experiences?

12. What do you consider the most challenging aspect of your SMEs?

13. Over time, how has your response to your SMEs changed?

14. Did you ever seek help to manage or cope with your SMEs?

15. What strategies have helped you manage or cope with your SMEs?

16. Anything else about your SMEs? 